sixty-three $(80 \%)$ dancers participated. Baseline scans were collected with follow-up scans at six weeks. The primary outcome was quantification of AT structure with Ultrasound Tissue Characteristics (UTC) (echo-type I-IV). Secondary outcomes of interest were clinical signs and symptoms gathered from a clinical examination, self-reported symptoms, VISA-A questionnaire and pain during single-legged heel raise. UTC has previously shown to have satisfactory reproducibility and validity. Separate multilevel linear and logistic regression models were performed including time and demographic variables as covariates.

Result From baseline to follow-up there was significant decrease in distribution of UTC echo-type I $(\beta=-3.6$, $\mathrm{p}=0.001 ; 95 \% \mathrm{CI}:-5.8 ;-1.5)$ with significant increase in echotype II $(\beta=3.2, p<0.0001 ; 95 \% \mathrm{CI}: 1.6 ; 4.8)$. Significant effects were also seen, of limb (type I+III) and gender (type I+II). No significant changes were found in clinical outcomes/clinical signs and symptoms.

Conclusion The cohort of ballet dancers showed significant UTC changes, mainly a reduction of echo-type I distribution after six-weeks pre-season period rehearsing Swan Lake ballet. No changes were found in clinical outcomes/clinical signs and symptoms. However, early structural changes seem important to follow longitudinally for potential planning of secondary prevention strategies.

\section{INCIDENCE OF SPORTS-RELATED INJURIES AND ILLNESSES IN PARALYMPIC ATHLETES - A PROSPECTIVE STUDY DURING 52 WEEKS}

${ }^{1}$ Kristina Fagher* ${ }^{*}$ 2,3 Örjan Dahlström, ${ }^{2}$ Jenny Jacobsson, ${ }^{2}$ Toomas Timpka, ${ }^{1,4}$ Jan Lexell. ${ }^{1}$ Department of Health Sciences, Rehabilitation Medicine Research Group, Lund University, Sweden; ${ }^{2}$ Department of Medical and Health Sciences, Athletics Research Center, Linköping University, Sweden; ${ }^{3}$ Department of Behavioural Sciences and Learning, Linköping University, Sweden; ${ }^{4}$ Department of Neuroscience, Rehabilitation Medicine, Uppsala University, Sweden

\subsection{6/bjsports-2019-scandinavianabs.27}

Introduction Sports-related injuries and illnesses in Paralympic sport (SRIIPS) is a growing concern, but knowledge about the etiology of SRIIPS is limited. The aim of this study was to prospectively assess the incidence and risk factors of SRIIPS among Swedish Paralympic athletes during 52 weeks.

Materials and methods 107 Paralympic athletes weekly reported SRIIPS in an eHealth application adapted to Paralympic athletes. Descriptive statistics and the Kaplan Meier method with corresponding Cox proportional hazards regression analysis $(p<0.05)$ was used to assess incidence, time to SRIIPS, survival probability and hazard ratio (HR).

Results The incidence of injuries and illnesses was 7.0/1000 and 9.5/1000 hours of sport exposure, respectively. For injury the survival probability was $31.8 \%$ and median time to injury was 19 weeks (95\% CI: 10.6-27.4). A higher risk to sustain an injury was noted among athletes; $\geq 30$ years (HR 1.6; 95\% CI: 1.01-2.53), with previous severe injury (HR 2.4; 95\% CI: 1.47-3.83), in team sports (HR 1.8; 95\% CI: 1.10-2.80) and males (HR 1.8; 95\% CI: 1.062.93). For illness, the survival probability was $23.4 \%$. Median time to illness was 9 weeks (95\% CI: 1.4-16.6). Athletes reporting an injury (HR 1.8; 95\% CI: 1.08-2.98) and participants in team sports (HR 1.6; 95\% CI: $1.05-$ 2.54) had a higher risk for illness.
Conclusion This is the first longitudinal long-term prospective study of SRIIPS. Males, older athletes, athletes in team sports and with previous incidents are particularly at risk for injuries and illnesses and should therefore be targets for preventive measures and future research.

\section{INCREASED HIP ADDUCTION DURING RUNNING IS ASSOCIATED WITH PATELLOFEMORAL PAIN AND DIFFERS BETWEEN MALES AND FEMALES: A CASE- CONTROL STUDY}

\begin{abstract}
${ }^{1,2}$ Bradley Neal ${ }^{*}{ }^{3,4}$ Christian Barton, ${ }^{1,5}$ Aleksandra Birn-Jeffrey, ${ }^{1,6}$ Dylan Morrissey. ${ }^{1}$ Sports and Exercise Medicine, Queen Mary University of Londoon, UK; ${ }^{2}$ Pure Sports Medicine, UK; ${ }^{3}$ La Trobe Sport and Exercise Medicine Research Centre, Australia; ${ }^{4}$ School of Allied Health, La Trobe University, Australia; ${ }^{5}$ School of Engineering and Materials Science, Queen Mary University of Londoon, UK; ${ }^{6}$ Physiotherapy Department, Barts Health NHS Trust, UK
\end{abstract}

\subsection{6/bjsports-2019-scandinavianabs.28}

Introduction Patellofemoral pain (PFP) is common amongst recreational runners and associated with altered running kinematics. However, it is currently unclear how sex may influence kinematic differences previously reported in runners with patellofemoral pain. This case-control study aimed to evaluate lower limb kinematics in males and females with and without patellofemoral pain during prolonged running.

Materials and methods Lower limb 3D kinematics were sampled in 20 runners with PFP (11 females, 9 males) and 20 asymptomatic runners (11 females, 9 males) during a $3 \mathrm{~km}$ treadmill run. Data were analysed when mean-pooled as mixed sex groups (PFP versus control) and as individual sex sub-groups.

Results Mixed-sex runners with PFP were found to have significantly greater peak hip adduction (mean difference $=4.9^{\circ}$, $\mathrm{d}=0.91, \quad 95 \%$ CI 1.4-8.2, $\mathrm{p}=0.01$ ) when compared to matched controls. Analyses for all other kinematic variables were non-significant. Females with PFP ran with greater peak hip adduction compared to female controls (mean difference $=6.6^{\circ}, \mathrm{p}=0.02, \mathrm{~F}=3.41,95 \%$ CI $0.4-12.8$ ), but not males with or without PFP. Analyses of sub-group comparisons for all other kinematic variables were non-significant.

Conclusion Differences in peak hip adduction between those with and without PFP during running appear to be driven by female participants, highlighting potentially different kinematic treatment targets for the individual sexes. Future research is encouraged to report lower limb kinematic variables in runners with PFP separately for males and females.

\section{IS TWO-DIMENSIONAL VIDEO A VALID AND RELIABLE MEASURE OF THREE-DIMENSIONAL KINEMATICS IN RUNNERS WITH PATELLOFEMORAL PAIN?}

\footnotetext{
1,2 Bradley Neal* ${ }^{1,2}$ Simon Lack, ${ }^{3,4}$ Christian Barton, ${ }^{1,5}$ Aleksandra Birn-Jeffrey ${ }^{1}$ Stuart Miller, ${ }^{1,6}$ Dylan Morrissey. ${ }^{1}$ Sports and Exercise Medicine, Queen Mary University of London, UK; ${ }^{2}$ Pure Sports Medicine, UK; ${ }^{3}$ La Trobe Sport and Exercise Medicine Research Centre, La Trobe University, Australia; ${ }^{4}$ School of Allied Health, La Trobe University, Australia; ${ }^{5}$ School of Engineering and Materials Science, Queen Mary University of London, UK; ${ }^{6}$ Physiotherapy Department, Queen Mary University of London, UK
}

\subsection{6/bjsports-2019-scandinavianabs.29}

Introduction Peak hip adduction (HADD) and knee flexion (KFLEX) during running are associated with patellofemoral pain (PFP) persistence, representing treatment targets. Clinical 
practice is lacking a validated, reliable tool with which to measure these kinematics. This study aimed to determine the accuracy of clinical gait analysis, by investigating concurrent validity, intra- and inter-rater reliability of two-dimensional (2D) video.

Materials and methods 21 participants with PFP were recruited (10 males, 11 females). Synchronised three-dimensional (3D) and 2D kinematic data were collected during over-ground running. 2D videos were analysed with the Hudl Technique application using a commercially available tablet (iPad). Single measure ICCs were calculated using a two-way mixed effects model with absolute agreement. 3D peak hip internal rotation (HIR) was investigated as a covariate with backward linear regression, using the $\mathrm{F}$ change statistic.

Results There was poor agreement between 3D and 2D measurement of peak HADD (ICC 0.06) and peak KFLEX (ICC 0.42). Moderate intra-rater reliability was identified for both variables (ICC 0.61-65). Inter-rater reliability for peak KFLEX was moderate (ICC 0.71), but was poor for peak HADD (ICC 0.31). 3D peak HIR did not significantly explain the identified poor agreement for either variable.

Conclusion Poor correlation between 3D kinematics and 2D video was identified for both variables in runners with PFP, despite acceptable intra-rater reliability. Investigation of software with increased precision is warranted, to improve the accuracy of $2 \mathrm{D}$ video predicting $3 \mathrm{D}$ kinematics in the clinical setting. Clinical gait analysis using the Hudl Technique application is not currently advocated.

\section{A NEW PROTOCOL FOR SONOGRAPHIC EVALUATION OF MID-PORTION ACHILLES TENDINOPATHY: A TEST RETEST INTRA AND INTER-RATER RELIABILITY STUDY}

${ }^{1}$ MSc Zubair Haleem*, ${ }^{2}$ Stephen Kelly, ${ }^{2}$ Dev Pyne, 'Simon Lack, ${ }^{1}$ Dylan Morrissey. ${ }^{1}$ Sports Exercise Medicine Dept - QMUL, Queen Mary University of London, UK; ${ }^{2}$ Rheumatology Dept, Mile End Hospital, UK

\subsection{6/bjsports-2019-scandinavianabs.30}

Introduction Achilles tendinopathy (AT) is common and recalcitrant. A new ultrasound Achilles tendon assessment protocol has been developed as part of a large RCT. Ultrasound scanning has considerable operator dependence hence this study was designed to investigate inter-rater and intra-rater reliability in symptomatic individuals.

Method Twenty-three participants (18 with AT, 10 female) were recruited and independently examined by two clinicians of varied experience. Measures of Achilles thickness, neovascularity, plantaris position and size, intratendinous tears (ITT) presence, fascia crura tear (FCT) presence, calcification, insertional elements and ultrasound provocation tests were recorded. Inter-rater and intra-rater reliability was assessed using Intraclass correlation coefficients (ICC), Standard error measurements (SEM) and minimal detectable changes (MDC) along with percentage agreements.

Results Excellent levels of inter- and intra-rater reliability were found for thickness (Intra-rater ICC 0.88-0.99, SEM 0.150.37 , Inter-rater ICC $0.87-0.98$ SEM 0.22-0.60), neovascularity (Intra-rater ICC $0.96-0.96$, SEM 0.28-0.40, Inter-rater ICC $0.91-0.94$ SEM $0.49-0.50$ ) and $100 \%$ agreement on insertional findings, ITT, FCT and US provocation tests. 67\%91\% agreement was found on presence, position and size of plantaris.
Conclusions The new protocol demonstrates excellent interand intra-rater reliability thus minimising operator dependence and is suitable for objective assessment. Further development of methods to identify the presence, position and size of the plantaris tendon is recommended.

\section{RISK FACTORS FOR LOWER-EXTREMITY INJURIES AMONG CONTEMPORARY DANCE STUDENTS}

${ }^{1}$ Christine van Seters, ${ }^{2,3}$ Rogier van Rijn*, ${ }^{1}$ Marienke van Middelkoop, ${ }^{2,3}$ Janine Stubbe. ${ }^{1}$ Department of General Practice, Erasmus MC University Medical Center, Netherlands; ${ }^{2}$ Codarts University of the Arts, Netherlands; ${ }^{3}$ Performing artist and Athlete Research Lab (PEARL), Netherlands

\subsection{6/bjsports-2019-scandinavianabs.31}

Introduction Contemporary dance students are at high risk for injuries; a yearly overall risk of $\geq 60 \%$ with lower-extremity injuries as the most predominant musculoskeletal injuries. Therefore, the objective was to determine whether student characteristics, lower-extremity kinematics, and strength are risk factors for sustaining lower-extremity injuries in preprofessional contemporary dancers.

Materials and methods A prospective cohort study with 45 1 year students of Bachelor Dance and Dance Teacher was set up. At the beginning of the academic year, injury history (only lower-extremity) and student characteristics (age, sex, educational program) were assessed using a questionnaire. Lower-extremity kinematics [single-leg squat(SLS)], strength (countermovement jump) and height and weight were measured during a physical performance test. Substantial lowerextremity injuries (main outcome) during the academic year were defined as any problems leading to moderate/severe reductions in training volume/performance, or complete inability to participate in dance at least once during follow-up as measured with the Oslo Sports Trauma Research Center (OSTRC) Questionnaire. Injuries were recorded on a monthly basis using a questionnaire. Analyses on leg-level were performed using generalized estimating equations(GEE) to test the associations between substantial lower-extremity injuries and potential risk factors.

Results The 1 year incidence of lower-extremity injuries was $82.2 \%$. Of these, $51.4 \%$ was a substantial lower-extremity injury. Multivariate analyses identified that ankle dorsiflexion during the SLS (OR1.25;95\% CI,1.03-1.52) was a risk factor for a substantial lower-extremity injury.

Conclusions The findings indicate that contemporary dance students are at high risk for LE injuries. Therefore, the identified risk factor (ankle dorsiflexion) should be considered for prevention purposes.

\section{TO INVESTIGATE FEASIBILITY OF A BANDCIZERTM DEVICE TO EVALUATE ADHERENCE TO HOME EXERCISE (HEP) FOR ADULTS WITH SHOULDER PAIN}

${ }^{1}$ Christina O'Connor, ${ }^{1}$ Jamie McNamara, ${ }^{1}$ Kathryn Fahy, ${ }^{1}$ Niamh Cleary, ${ }^{1}$ Paul Deering,

${ }^{1,2}$ Karen McCreesh*. ${ }^{1}$ School of Allied Health, University of Limerick, Ireland; ${ }^{2}$ Health Research Institute, University of Limerick, Ireland

\subsection{6/bjsports-2019-scandinavianabs.32}

Introduction Exercise is an effective intervention for rotator cuff related shoulder pain (RCRSP). Self-report methods of exercise adherence are subject to bias. The BandCizer ${ }^{\mathrm{TM}}$ is a 